\title{
Mixing is a double-edged sword: the grouping and segregation error on the assayer's bench
}

\author{
R.C.A. Minnitt, ${ }^{a}$ S.C. Dominy ${ }^{b}$ and K.H. Esbensen ${ }^{c}$ \\ avisiting Emeritus Professor, School of Mining Engineering, University of the Witwatersrand, Private Bag 3, WITS, \\ 2050, Johannesburg, South Africa \\ bisiting Associate Professor, Minerals Engineering Research Group, Camborne School of Mines, University of \\ Exeter, Cornwall, Penryn TR10 9FE, UK; Adjunct Professor, Department of Mining and Minerals Engineering, \\ Western Australian School of Mines, Curtin University, Bentley, WA 6102, Australia \\ ${ }^{c}$ Consultant, Copenhagen $\varnothing$, Denmark, www.kheconsult.com. Adjunct professor, Aalborg University (AAU), \\ Denmark; Adjunct professor, Geological Survey of Denmark and Greenland (GEUS); Professeur associé, Université \\ du Québec à Chicoutimi (UQAC), Québec; Guest professor University of Southeast Norway (USN); Guest \\ professor (2018) Recinto Universitario de Mayaguez, Puerto Rico
}

Even though our example is only taken from the assaying laboratory, when engaged in sub-sampling it is, at all scales, imperative to counteract the influence of material heterogeneity. Mixing, shaking and other agitation traditions is a critical success factor for reliable analytical aliquot extraction-but mixing can be a double-edged sword, as is demonstrated forcefully here with experimental evidence. Mixing leads to different results, a.o. depending on grain size contrasts (the case of gold grains in a quartz mix given here is an extreme graphical example). Mixing is also a function of more-or-less substantiated anecdotal "beliefs". But it is always necessary to base laboratory mixing protocols on a full Theory of Sampling understanding of the many practical manifestations of heterogeneity, and how to reduce its influence to the fullest degree possible. Mixing is only a panacea for those in the know ...

\section{Introduction}

The Grouping and Segregation (GSE) error is a fundamental member of the seven sampling errors defined and described by Pierre Gy (eight sampling errors if one includes the Increment Weighing Error, which Gy often considered "easy" to control). GSE is related to the combined effects of gravity interacting with the characteristics of the material being

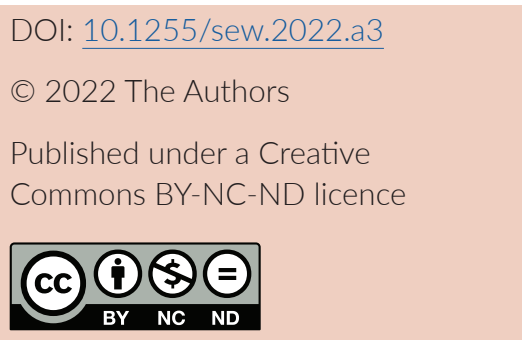

sampled, most commonly arising when the spatial distribution of fragments relative to one another are not uniform and when particulate materials are moved, transported or stacked in stockpiles. GSEs arise due to a static lot spatial heterogeneity and/or to significant input of kinetic energy to a material system. The principal factors involved in inducing GSE effects concern differences in fragment size, density, shape and variation in moisture content across particulate material lots being sampled a.o. affecting particle wettability.

We present experiments focused on traditional, strongly held notions on how to "homogenise" a powder batch on the assayer's laboratory bench. There are significant lessons to be learned regarding the universal Sampling Unit Operation: Mixing.

An example directed at the strongly opposing points of view regarding inducement of GSEs supposed to occur during aliquot preparation by the so-called "powder-rolling" method. Here pulverised rock powders (often ore/ gangue mixtures) are alternately flicked, or "rolled", from one corner to another on flexible plastic sheeting or wax-wrap paper. Assayers strongly believe that this is an ideal technique for homogenising the pulverised material before selection a 30-50 g aliquot for analysis, whereas many purist sampling experts and practitioners are of the opinion that rolling the pulverised material from side-to-side will unavoidably induce unwanted, and 
uncontrolled, segregation of fine and significantly denser particles in the powdered material (gold particles, Pt and other heavy ore minerals). Assayers have long considered this type of "rolling" as an essential means of homogenising pulp before aliquot preparation, especially in the gold mining industry. The present experimentation and observations using $\mathrm{X}$-ray tomography has confirmed this belief to some degree, but it was also found that when shaking and rolling highdensity contrast materials, the ensuing segregation depends on the intended outcome; occasionally powders containing gold grains that are shaken with the purpose of homogenising the mixture can end up actually inducing segregation or grouping. Below we try to elucidate and make sense of these phenomena.

\section{In the assaying laboratory}

Typical workflows in many analytical laboratories include attempts by the assayer to ensure that the pulverised powder from which the final aliquot to be extracted is thoroughly homogenised. Such procedures in the fire assay process are not uncommon and have been observed in numerous assay laboratories internationally. One of the most common methods to achieve this end is shown in Figure 1 which is documented from two laboratories, one in Suriname and one in Brazil.

In each case between $200 \mathrm{~g}$ and $400 \mathrm{~g}$ of pulverised powder, usually $95 \%$ passing $75 \mu \mathrm{m}$, is placed on a sturdy plastic sheet with the assayer rolling, or "flicking" the powder batch diagonally back and forth across the sheet for about 100 s. Local procedures differ from assay lab to the next in detail, but the general principle is identical.

However, many sampling practitioners frown on this rolling practise, because it is believed that such actions actually induce GSE effects in the batch just before the analytical aliquot is extracted. The belief is strong, but repeatable scientific evidence to support this notion appears to be unavailable. This practice is nevertheless extensively performed in many laboratories visited by the authors, which carries enough concern that this procedure should be a topic of empirical research. A further strong belief, correct or misplaced, is that a traditional riffle splitter also induces GSE, especially when sub-sampling particulate materials with target analytes, such as gold, occurring as individual, or residing in, highdensity grains. These two popular beliefs are investigated in the present practical investigation.

\section{Experimental design; materials}

An experiment to investigate the degree to which GSE can be induced in pulverised assay powders by rolling was designed and appropriate equipment set up. Approximately 300 small gold grains ranging in size from $100 \mu \mathrm{m}$ to $300 \mu \mathrm{m}$ ("fine-grained gold particles") shown in Figure 2(a), were mixed in $150 \mathrm{~g}$ of finely ground quartz (95\% passing $150 \mu \mathrm{m}$ ), as shown in Figure 2(b), and placed in a plastic jam jar containing a finely ground quartz matrix. This mixture constitutes the experimental batch to be homogenised by alternative techniques before extraction of one or more analytical aliquots.

\section{Shaking pulverised fire assay powders}

The plastic batch container with gold grains/quartz powder was first
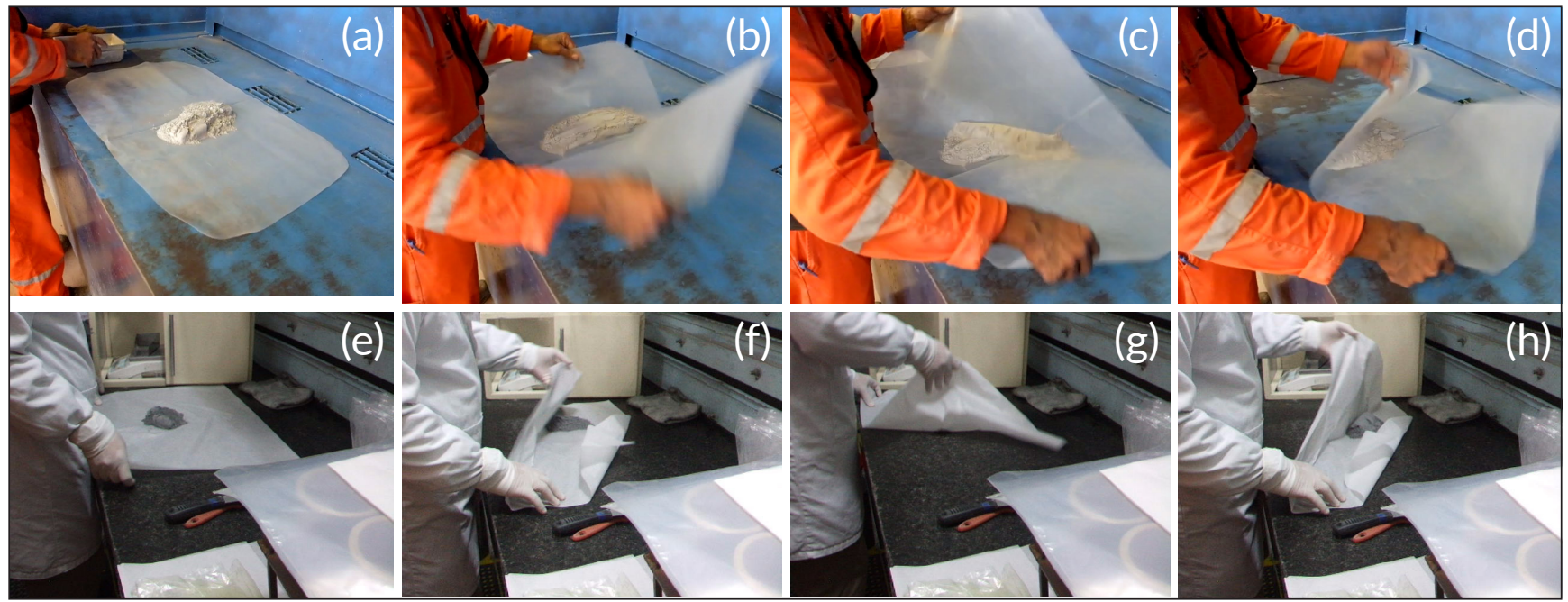

Figure 1. Pulverised rock powder for fire assay is "homogenised" by rolling or flicking a powder batch on a flexible sheet immediately before aliquot extraction. (a-d) Rolling powder from side-to-side operation performed 40 times. (e-h) Same operation, but here only performed 20 times. Still photos extracted from video recordings. Illustration copyright: RCAM teaching collection; used with permission. 

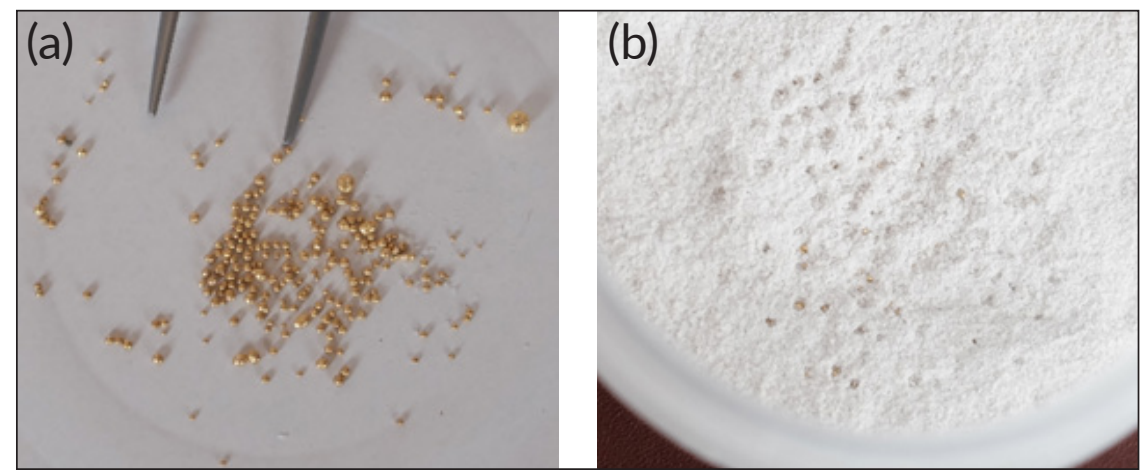

Figure 2. a) Approximately 300 gold grains, ranging in size from $100 \mu \mathrm{m}$ to $300 \mu \mathrm{m}$, and b) finely ground quartz matrix showing gold grains on surface before mixing. Illustration copyright: RCAM teaching collection; used with permission.

thoroughly shaken in an attempt to mix the contents, as per universal common belief that shaking $=$ good mixing. The content was then $\mathrm{X}$-ray scanned, ${ }^{1}$ showing a fairly even distribution of gold grains as seen in the vertical cross-section of the container as shown in Figure 3(a).

The container was subsequently vigorously agitated by hand in a deliberate attempt to induce segregation, carefully making sure that there was no vertical movement component, only a sideways action. The container was again scanned, and the resulting distribution is shown in the cross-section of the container as Figure 3(b). Clearly this attempt to induce GSE of the highdensity gold grains in the quartz powder was successful, as can be seen by the grouping of grains at the base and in horizontal layers on the right-hand side of the container [Figure 3(b)].

Clearly grouping and segregation does take place but considering the degree to which the powder was agitated, for approximately $120 \mathrm{~s}$, and given the density of gold $19.3 \mathrm{~g} \mathrm{~cm}^{-3}$ vs the density of the quartz powder, about $2.0 \mathrm{~g} \mathrm{~cm}^{-3}$, a greater degree of segregation was perhaps expected. However, as there was no quantitative way of meaningfully measuring the degree of in-mixture agitation, repetitions of the experiment would in all likelihood produce different results, i.e. different degrees of grouping and segregation. The reader is welcome to snap into action to perform a more systematic series of experiments of this kind; this topic is eminently suited for a minor academic project.

\section{Rolling pulverised fire assay batches}

The fine gold grains and pulverised quartz powder was again thoroughly mixed before being placed on a clean sheet of tracing paper. Alternate diagonal corners of the sheet were lifted several times, "rolling" the powder from one corner to the other, carefully duplicating the rolling actions seen in many mine assay laboratories. This was a deliberate attempt to induce segregation of the gold grains (if such is one's original belief)-or, contrarily, to mix the batch well. Which was it?

The processed powder batch was then carefully placed in a plastic bag, which was immediately vacuum sealed so that the spatial distribution of the gold and quartz grains achieved by rolling could not be disturbed by any further handling, Figure 4(a). This vacuum sealed bag was placed in an X-ray tomography unit housed in the Palaeosciences Unit at Wits University, and scanned [Figure 4(b)].

This experiment was repeated three times using increasing levels of vigour to investigate whether the intensity of the rolling action made discernible differences to the distribution of the gold grains, or not.

These three rolling experiments show no indications of strong segregation of fine gold grains despite the gold grains used in

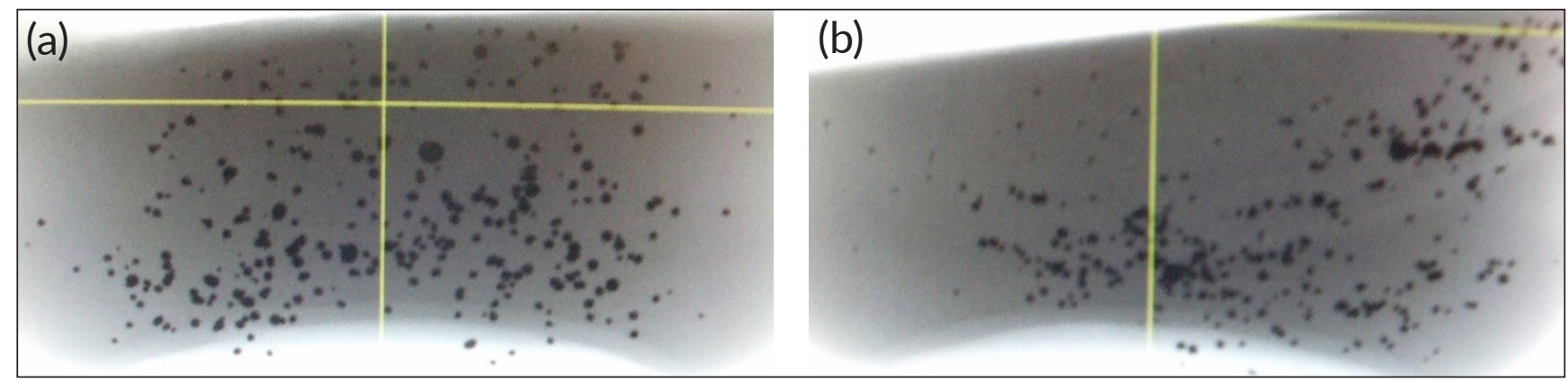

Figure 3. X-ray scans (vertical cross-sections of container). (a) Evenly distributed gold grains in quartz powder after thorough initial mixing. (b) Significant grouping of gold grains in layers (right-hand side) and near the base of the container (segregation) after sustained horizontal agitation of the container. For X-ray tomography details, see method description associated with Figure 4. Illustration copyright: RCAM teaching collection; used with permission. 


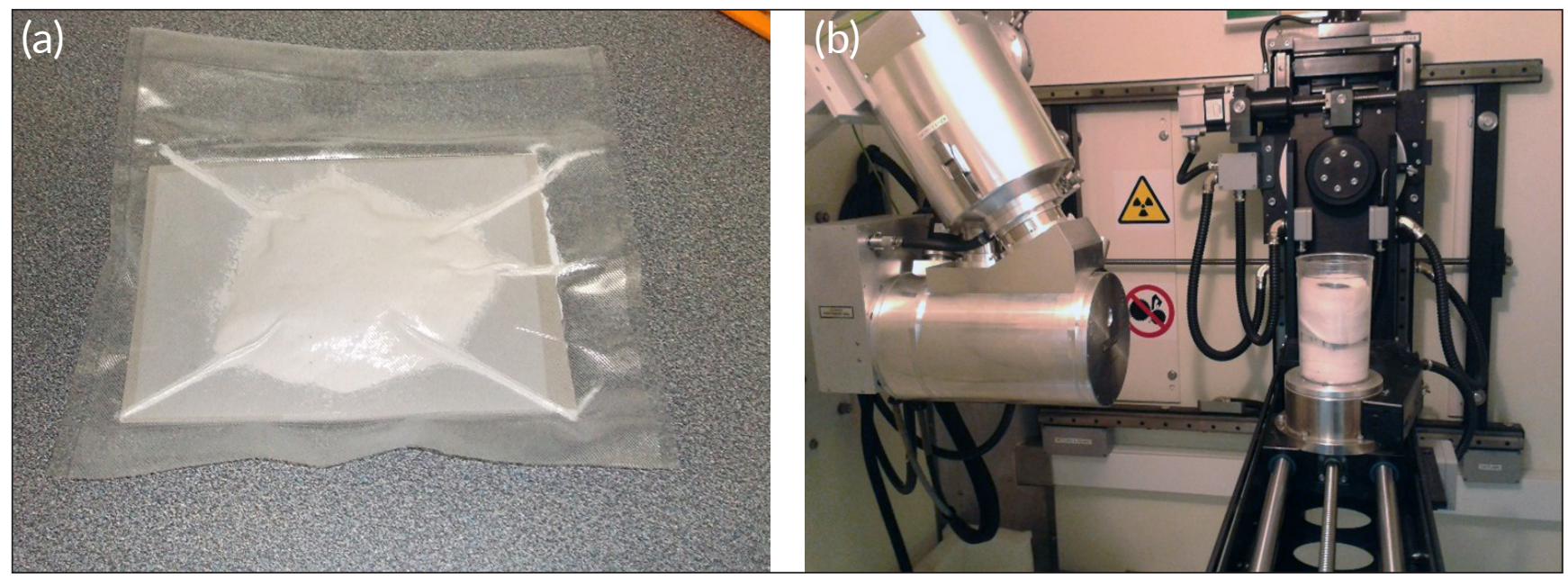

Figure 4. (a) Vacuum sealed plastic bag containing a rolled quartz-gold grain mixture on a cardboard sheet, see Figure 5. (b) Analytical setup of X-ray tomography instrument housed in the Palaeosciences Unit, Wits University; this shows scanning of the original mixture plastic batch container, cf. Figures 2 and 3. Illustration copyright: RCAM teaching collection; used with permission.

the experiment being the highest density of practical interest in the mining and minerals processing industry.

\section{Discussion of induced segregation experiments}

Shaking experiments

The results of the first simple examples of shaking and rolling of fine gold grain-bearing powders suggest that, to some extent, the degree of segregation depends on the intended outcome. Powders containing fine gold grains that are shaken with the purpose of homogenising the mixture, seem to be able to achieve that end [Figure 3(a)], whereas shaking with the clear intention of inducing segregation or grouping, can also be successful [Figure 3(b)]. (See Brazil nut effect box below.)

\section{Rolling experiments}

Comparative scanned X-ray results for the three rolling experiments are shown in Figure 5. The experiment was carried out three times using increasing levels of vigour (left to right), to elucidate whether the intensity of the rolling action made discernible differences to the distribution of the gold grains. In each case the limit of the flattened volume of powder in the vacuum sealed bag is shown by the dotted black perimeter, with individual gold grains in the powdered lot represented by the black dots.

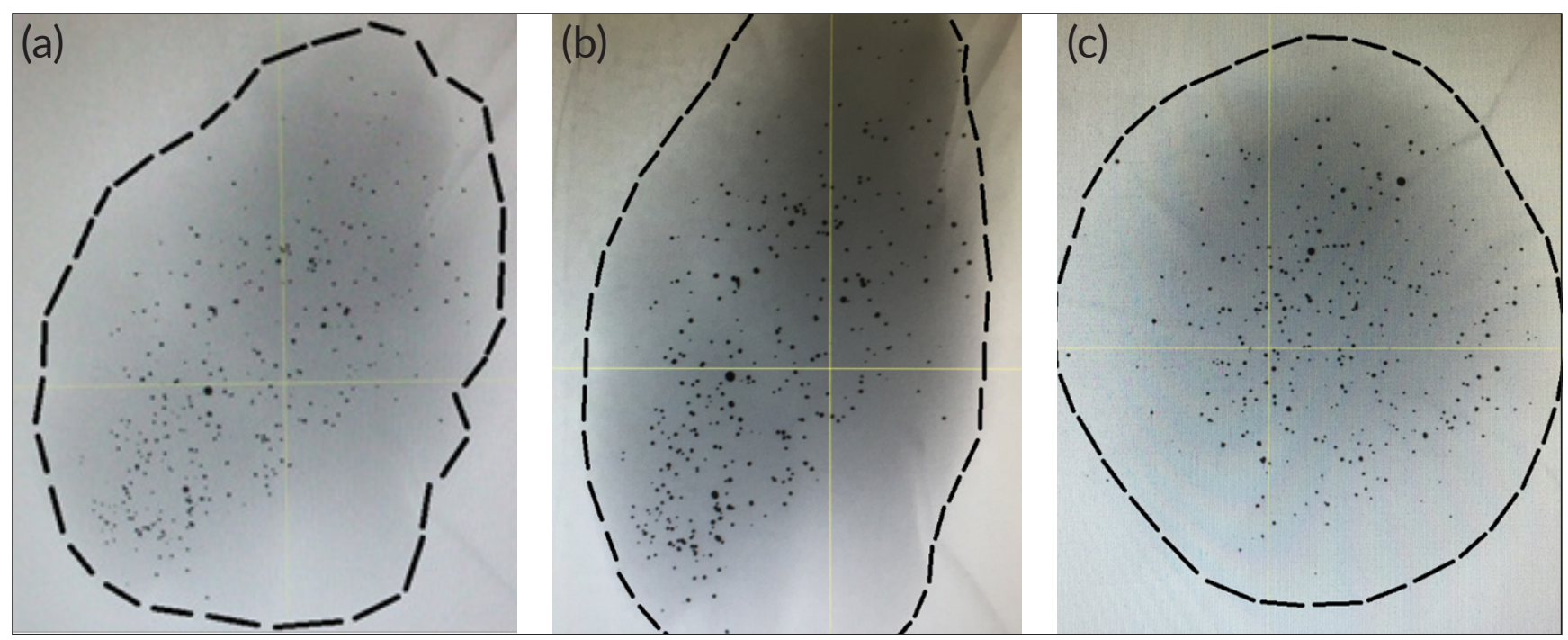

Figure 5. X-ray scanning images representing increasing rolling intensities. (a) Gold grains in the first experiment (lowest rolling intensity). (b) Distribution of gold grains for the second experiment. (c) Gold grain distribution for the third experiment (highest rolling intensity). Illustration copyright: RCAM teaching collection; used with permission. 


\section{SAMUPLINECOLLUMAN}

The distribution of fine gold grains suggests that there is no segregation or grouping despite the thorough rolling, and despite the gold-quartz grains used represent of the highest density contrast mixed material representing relevant fine gold mineralisation ore types and similar-or fully liberated ore types of the same composition. This fact makes inferences from this simple experiment of a more general scope.

It can, therefore, be concluded that the rolling of assay powders as carried out in many assay laboratories, does not necessarily induce segregation or grouping of fine-grained, high-density target analytes, suggesting there are few grounds for terminating this process in analytical laboratories. Though, as always, care is required with duplicate or triplicate representative fire assaying undertaken on the pulp as part of the QC process to monitor variability.

\section{Scooping of aliquots from fire assay batches}

Thus, the dominating evidence shows that rolling of fire assay batches does not induce compromising GSE, but there is also evidence to suggest that "mixingshaking" in jars can be a doubleedged sword. Of key importance, however: subsequent scooping of powder from a batch (however "well mixed"), the next step in the preparation of an aliquot for fire assay, can easily be impacted by significant GSE effects. If so, what is gained on the swings is lost on the roundabouts.

Aliquot preparation by scooping from a powdered lot is even more common in assay laboratories than is the rolling procedure to "homogenise" a powder mixture. Examples of this procedure are shown again from two laboratories in Figure 6.

In the upper panel, a scoop is dipped into the powder and a quantity is extracted as the aliquot. Note that the "driving force" for this type of extraction is the mass to be realised for analysis. The analyst is wholly focused on measuring an exact amount, say $20 \mathrm{~g}, 30 \mathrm{~g}$ or $50 \mathrm{~g}$, and then adds whatever reagents are required to the assay crucible. In the lower panel of Figure 6 the assayer is aiming at a relatively large amount of powder by scooping an approximate amount and determine the precise aliquot mass afterwards by weighing. But the salient point is that these are both mass-driven grab sampling approaches, justified by assuming that mixing will always assure a representative aliquot extractionby any preferred method. Although very often met with in practice in the world's analytical laboratories, this is in fact a seriously flawed assumption which is better always questioned. There are many illreflected mixing approaches in use that do not secure a representative
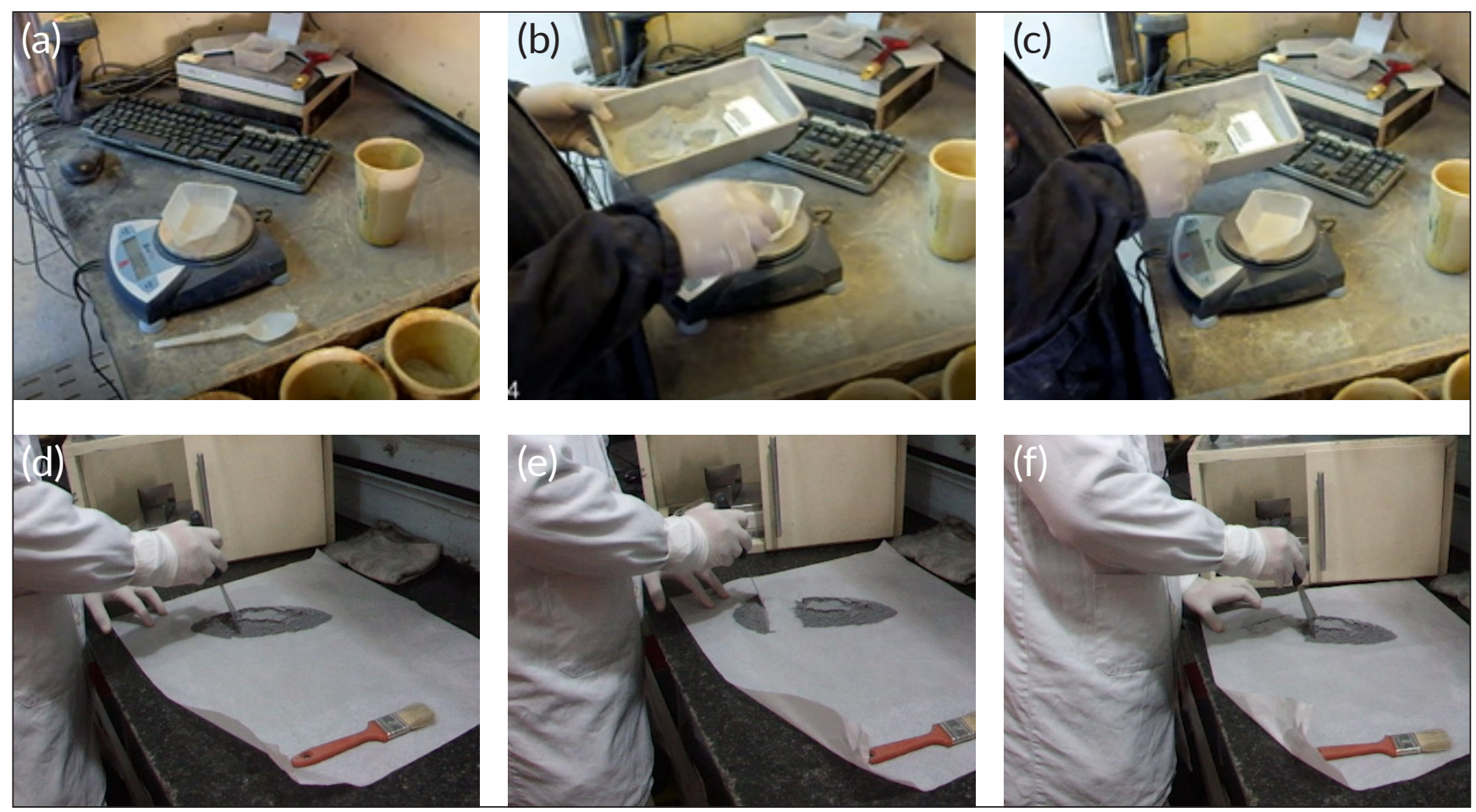

Figure 6. (a-c) Sequence of steps showing mass-driven scooping of powder from a pulverised batch in the creation of a fire assay aliquot. $(\mathrm{d}-\mathrm{f})$ Splitting of a batch into three more-or-less equal aliquots. However, both approaches are flawed w.r.t. counteracting the empirical heterogeneity encountered in the pre-aliquoting laboratory batch. Obviously "mixing" helps but there is no guarantee of achieving sufficient mixing in and of itself. Illustration copyright: RCAM teaching collection; used with permission. 


\section{Brazil nut effect}

The famous "Brazil nut effect" example of marked segregation results from agitation with the deliberate objective of obtaining the exact opposite effect: mixing. "Have you ever noticed that the dried fruits or nuts in your breakfast cereal are not evenly spread out inside the box-or that in a container of mixed nuts, Brazil nuts gather at the top? This phenomenon is commonly called the "Brazil nut effect", and the science behind it is surprisingly complex and farreaching. This situation can be a nuisance when you want to fill silos, bags or bins with different types of materials. But it can also be used to our advantage ..." https://www. scientificamerican.com/article/ all-mixed-up-discover-the-brazilnut-effect/

This Brazil nut effect points to the other major factor in inducing GSE due to agitation, that of contrasting grain size(s), e.g. see https://www. sciencenewsforstudents.org/ article/brazil-nut-effect-mixednuts-xray-scan-physics

aliquot (thorough mixing or not), as explained in detail in Reference 2.

These types of scooping at laboratory batch scale as shown in Figure 6 are, therefore, unacceptable in the context of the Theory of Sampling (TOS) and should emphatically be terminated. Efforts should instead be directed at universally substituting all types of small-scale spatula grab sampling by proper, "correct" aliquot extraction, preferentially using a bench-scale rotary splitter or a micro-splitter specially designed for sub-sampling very small powder masses, Figure 7; see also Chapters 12 and 13 in Reference 2.

This denouncement of grab sampling-at any scale-is regardless of the state of realised mixing of the batch material, which may
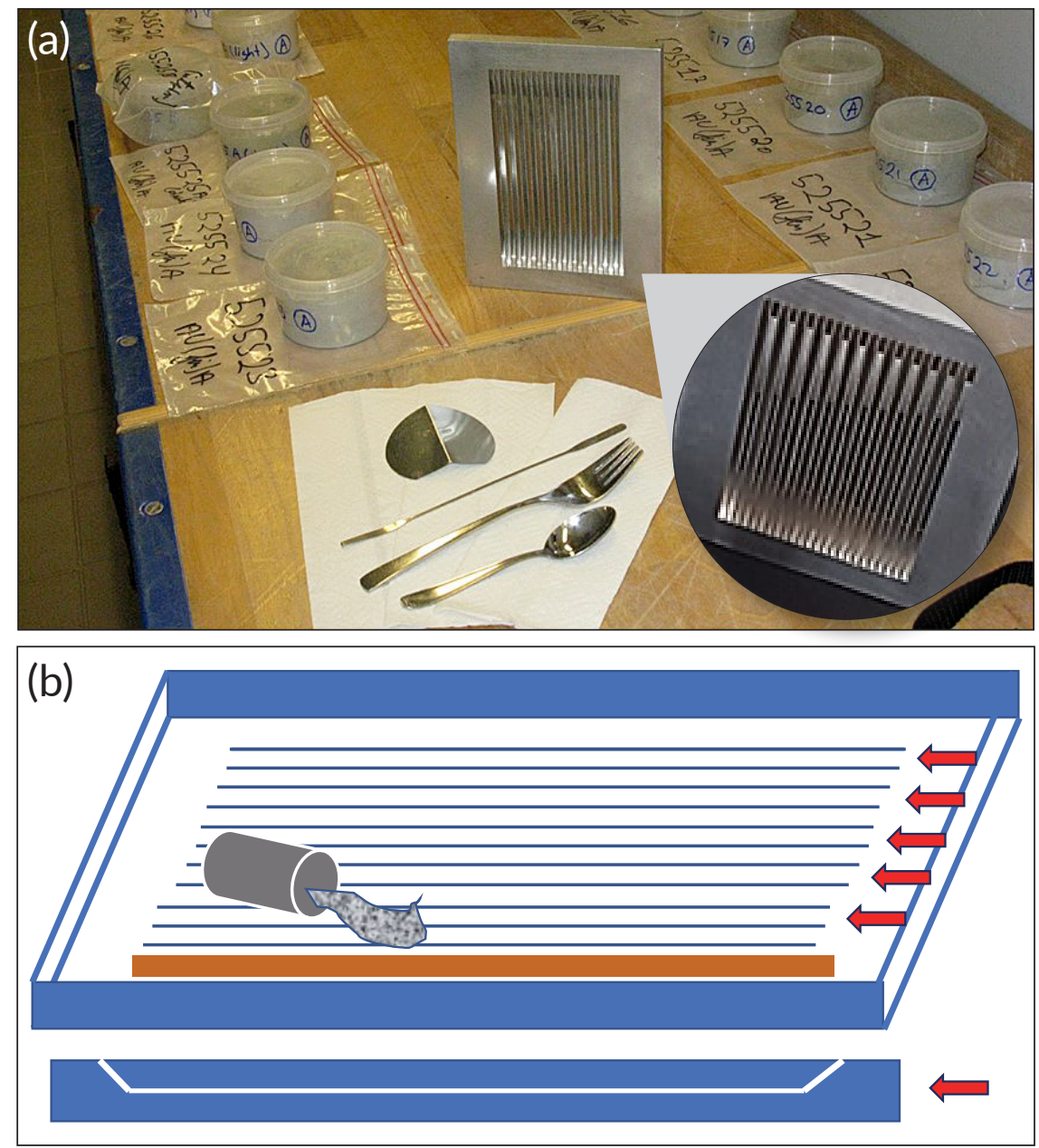

Figure 7. (a) Foreground: a variety of common but misguided laboratory equipment with which to perform sub-sampling operations on the laboratory bench: fork (a mixing tool) and spoon, spatula a.o. for extracting analytical aliquots, none of which respect the representativity demands from the TOS. Background: the famous, but little known, "Ingamells micro-splitter", an ingenious implement invented by Charles Oliver Ingamells (1916-1994), ${ }^{3}$ for use in sub-sampling miniscule amounts of reference materials for microprobe analysis. This splitter is preferred for all laststage mass-reduction of fine powders and similar because it is guaranteed to be representative cf. the spatula, which still dominates in very nearly all the world's analytical laboratories. (b) Schematic of the function of the Ingamells micro-splitter, a stationary riffle splitter machined from a solid aluminium frame with an even number of alternating chutes (riffles). Every second chute is machined to penetrate fully through the frame-with the complementary set only machined to $2 / 3$ depth. The chutes that go all the way through allow $50 \%$ (vol/vol) of the poured mass to pass completely through the frame and to become the reject. While the intercepted material staying in the $2 / 3$ depth chute grooves becomes the $50 \%$ split off subsample. The non-penetrating chutes have a slanted (or curved) end configuration to facilitate "sliding out" the intercepted (sampled) material. Ingamells micro-splitter courtesy the estate of Oliver Ingamells through kind donation from Francis Pitard. Illustrations copyright: KHEC teaching collection; reproduced with permission. 


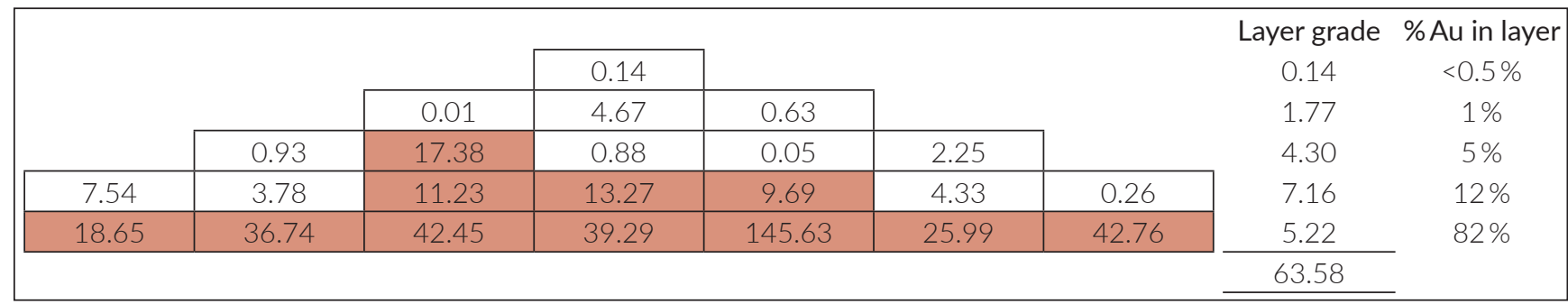

Figure 8. Cross-section transect through a pulp pile showing 23 extracted assays with marked "segregated" gold at the lower part of pile (red highlights). The global grade of the $2045 \mathrm{~g}$ pile is $29.1 \mathrm{~g} \mathrm{t}^{-1}$ Au based on the complete total of 42 fire assays. The distribution shown is approximate relative to the challenge of complete 3D pile sampling, but the direction of this transect was chosen at random.

\begin{tabular}{|c|c|c|c|c|c|c|c|c|}
\hline & & & & & & & Layer grade & \% Au in layer \\
\hline & & & 0.67 & & & & 0.67 & $1 \%$ \\
\hline & & 0.04 & 1.25 & 16.1 & & & 5.80 & $29 \%$ \\
\hline & 0.75 & 0.95 & 12.4 & 0.05 & 0.02 & & 2.83 & $23 \%$ \\
\hline 0.09 & 0.78 & 1.07 & 1.42 & 0.98 & 0.34 & 0.27 & 0.71 & $8 \%$ \\
\hline 0.86 & 3.98 & 0.04 & 0.39 & 1.45 & 15.7 & 0.73 & 3.31 & $38 \%$ \\
\hline & & & & & & & 13.31 & \\
\hline
\end{tabular}

Figure 9. Cross-section through the centre of another pulp pile showing 23 assays taken, and minor "segregated" gold at the lower part of pile (red highlight), here with some higher grades towards the top of the pile (orange). The global grade of the $2135 \mathrm{~g}$ pile is $3.7 \mathrm{~g} \mathrm{t}^{-1}$ Au based on an exhaustive 43 fire assays. The distribution shown is approximate relative to the challenge of complete 3D pile sampling, but the direction of this transect was chosen at random.

be anything from unchecked belief only to fit-for-purpose acceptable depending on the informed mixing operation(s) deployed. The point here is that it is not the particular method in and of itself that can further a guarantee for sufficient mixing-only a full understanding of the various, perhaps competing methods and their empirical substantiation, is an acceptable basis for laboratory mixing protocols.

Hence our humble, first foray experiments disclosed here.

\section{Industrial case study: coarse gold grains}

Dominy ${ }^{4}$ presents a case study from an underground gold vein mining operation that was known to contain coarse gold particles up to $1.2 \mathrm{~mm}$ in size-contrast with Figure 2. It was observed that pulp duplicates displayed very poor precision ( $\pm 66 \%)$. A series of tests was undertaken on $2 \mathrm{~kg}$ pulp batches, where the batch pile was mixed (similar process to that seen in Figure 1) and 40-45 consecutive 40-50 g subsamples were extracted for fire assay. The variability was remarkably high, and in one instance the range between the minimum and maximum analytical results was $500 \mathrm{~g} \mathrm{t}^{-1} \mathrm{Au}$.

The tests were undertaken by grabbing consecutive $50 \mathrm{~g}$ pulp fire assay charges from the roughly conical pulp pile. This was done to completion, i.e. until the pile was totally consumed. It was found that the highest grades were generally hosted in the lower $10 \mathrm{~mm}$ of the pile. In one case, the lower section contained $82 \%$ of the sample gold (Figure 8). Any $50 \mathrm{~g}$ assay charge taken from the upper part of the pile thus severely understated the sample grade.

Figure 9 shows a section through a lower-grade pulp pile $\left(3.7 \mathrm{gt}^{-1} \mathrm{Au}\right.$ in which minimal coarse gold was observed in the primary sample), where there is some potential segregation at the base of the pile, but also some higher grades towards the top. These may reflect "reverse segregation" where gold particles segregate upwards during "mixing" (see Brazil nut effect box), irregularly "enriching" the upper part of the pulp pile.

These findings confirm that routine pulps can be highly heterogeneous due to poor comminution of gold particles during pulverisation and that coarse gold was generally segregating to the bottom of the pile during "mat rolling".

Other test work confirmed that primary $2-3 \mathrm{~kg}$ mine channel samples had visible gold sizing between $500 \mu \mathrm{m}$ and $1500 \mu \mathrm{m}$, but after being pulverised still contained gold sizing between $250 \mu \mathrm{m}$ and $750 \mu \mathrm{m}$. The sample presented in Figure 8 was the most extreme showing severe "base" segregation. The other nine test pulp samples displayed varying base segregation, with two samples showing some upper-level segregation relating to the "Brazil nut effect" (Figure 9). In all of these test cases, the upper part of the pile where the scoop 
would have been routinely taken were either depleted (Figure 8) or enriched in gold (Figure 9).

Samples showing a grade of $>15 \mathrm{~g} \mathrm{t}^{-1} \mathrm{Au}$, generally displayed some segregation, more often at the pile base. This was confirmed by other test work extracting one singular scoop from the pile with the remnant batch assayed in total via screen fire assay. The full screen fire assayed lots were generally $10-50 \%$ higher grade than the single scooped values.

Grades between $12 \mathrm{gt}^{-1} \mathrm{Au}$ and $24 \mathrm{gt}^{-1}$ Au could indicate the presence of a single $500 \mu \mathrm{m}$ gold particle within the fire assay charge, dependent upon the gold particle shape (e.g. from flake to sub-spherical). Grades below $1 \mathrm{gt}^{-1} \mathrm{Au}$ will be dominated by $<150 \mu \mathrm{m}$ gold. The $<0.1 \mathrm{gt}^{-1} \mathrm{Au}$ grades will be dominated by $<75 \mu \mathrm{m}$ gold. These assertations are supported by mineralogical and screen fire assay test work.

\section{Discussion: coarse gold particles}

The key phenomenon exposed above is that coarse gold needs to be treated differently from fine-grained gold. ${ }^{5-7}$ Pulps bearing coarse liberated gold cannot be homogenised; GSE effects will then be highly problematic and proper protocols must be set up in the laboratory. In the present case, a screen fire assay was introduced to account for coarse gold, along with improved laboratory procedures and better staff training. The $2-3 \mathrm{~kg}$ pulp was split using a TOS-compliant riffle splitter to $1 \mathrm{~kg}$ for screen fire assay. QA/ QC protocols were introduced, particularly covering equipment cleaning and contamination monitoring as well.

The key lesson from this work is that as soon as coarse gold is liberated in a pulp, the best course of action is to analyse the entire lot via a bulk assay method (e.g. screen fire assay or LeachWELL). Alternatively, the practitioner should look at technology such as PhotonAssay, which can assay a "bulk" sample (easily from $500 \mathrm{~g}$ to $5000 \mathrm{~g}$ or more) of crushed material. $^{8}$

The traditional sampling paradigm of crush, split, pulverise and fire assay (30-50 g charge size) is flawed in the presence of coarse gold (certainly > $150 \mu \mathrm{m}$ in the pulp), particularly when the assay charge is grabbed or scooped from the pulp.' Bulk assay methods offer the only correct approach. Good preparation equipment hygiene is required, with barren flushes of crushing and pulverising equipment undertaken regularly and assayed. ${ }^{5,9}$

Proper ore characterisation is required to ensure that sample collection, preparation and assay protocols are fit-for-purpose and representative. ${ }^{2,7}$ In addition, empirical test work on pulps is required to determine and calibrate protocols in the presence of coarse gold after pulverising.

\section{Mixing: a highly variable, hidden success factor}

Even though here only shown by the assaying laboratory, at all scales and for all types of mixed materials, it is imperative to counteract the influence from material heterogeneity optimally, thereby reducing the influence from GSE to the fullest degree possible. The principal effects elucidated here will manifest themselves to different degrees according to the materials treated etc., but they never go away. Mixing, shaking and other agitation traditions are critical, often unknown or ill-reflected hidden success factors for reliable analytical performance-but un-reflected mixing blindly used as a universal agent is a double-edged sword that can lead to major surprises.

Sampling along the full lotto-aliquot pathway, certainly including the laboratory realm of sub-sampling of particulate materials by scooping or by grabbing (scale doesn't really matter) ${ }^{2}$ will always result in potentially compromised aliquot representativity vis-à-vis the primary lot, stock or batch, Figures 6-9. With the presence of coarse gold (>>100 $\mu \mathrm{m}$ ) in the pulp, extreme care is required if using mixing to "homogenise" prior to splitting by scooping or grabbing. Significant GSE is possible when $>500 \mu \mathrm{m}$ gold is present, which will have a highly detrimental impact on the quality of a scooped or grabbed assay aliquot. A perhaps counterintuitive "reverse segregation" can also occur. ${ }^{10}$ The imperative here must be (1) to take the entire pulp batch for bulk analysis or (2) when sub-sampling is necessary, then only use a TOS-compliant riffle or RSD splitter. Grabbing or scooping is not acceptable.

It is the degree of optimal mixing before final extraction of aliquots that matters most-together with adverse grain size contrasts, all the world's more-or-less "smartly designed" extraction implements notwithstanding. It is also of critical importance to seek information of the de facto grain sizes involved, especially for analytes occurring as, or embedded in, coarse particles that cannot be ground down by standard equipment; gold is the prime mineralogical example (also of extreme monetary value). Belief, blind trust and use of standard mixing procedures and equipment is no guarantee for sub-sampling representativity-a minimum of TOS competence is always necessary.

\section{Acknowledgements}

The authors gratefully acknowledge the help and assistance of Mr Kudakwashe Jakata, Scanning Manager, in the Centre of Excellence in Palaeosciences at the University of the Witwatersrand in South Africa with the scanning of the experimental materials used in this paper. 


\section{References}

1. R.C.A. Minnitt, J. Jashasvili, G. Gilchrist and S.C. Dominy, "Quantifying segregation of minerals and metals in particulate materials using computed X-ray tomography and variography", in Proceedings of the World Conference on Sampling and Blending 2017. Australasian Institute of Mining and Metallurgy: Melbourne, Australia, pp. 171-182 (2017).

2. K.H. Esbensen, Introduction to the Theory and Practice of Sampling. IM Publications Open (2020). https://doi. org/10.1255/978-1-90671529-8

3. F. Pitard, "The legacy of Charles Oliver Ingamells (1916-1994)", TOS Forum 10, 24 (2020). https://doi.org/10.1255/ tosf.123

4. S.C. Dominy, "It does not matter what is wrong when applying TOS: it is money out of the window", Spectrosc. Europe
33(7), 41-42 (2021). https://doi. org/10.1255/sew.2021.a41

5. S.C. Dominy and J.S. Petersen, "Sampling coarse gold-bearing mineralisation-developing effective protocols and a case study from the Nalunaq mine, Southern Greenland", in Proceedings of the World Conference on Sampling and Blending 2005. Australasian Institute of Mining and Metallurgy: Melbourne, Australia, pp. 151-165 (2005).

6. S.C. Dominy, "Importance of good sampling practice throughout the gold mine value chain", Mining Tech. 125(3), 129-141 (2016). https://doi.org/10.1179 /1743286315Y.0000000028

7. S.C. Dominy, I.M. Platten, H.J. Glass, S. Purevgerel and B.W. Cuffley, "Determination of gold particle characteristics for sampling protocol optimisation", Minerals 11, 1109 (2020). https://doi.org/10.3390/ min11101109
8. C.D. Tremblay, J. Tickner, D. Treasure, A. Oteri and G. Wheeler, "PhotonAssay - efficient and bulk gold analysis in the modern world", in Proceedings of the International Mining Geology Conference 2019. Australasian Institute of Mining and Metallurgy, Melbourne, Australia, pp. 88-98 (2019).

9. S.C. Dominy. "Sampling coarse gold-bearing mineralisation - developing effective protocols and a case study from the Ballarat mine, Australia", in Proceedings of the World Conference on Sampling and Blending 2017. Australasian Institute of Mining and Metallurgy, Melbourne, Australia, pp. 171-182 (2017).

10.S.C. Dominy, A.E. Annels and A.G. Royle, Sampling and Estimation of Gold Deposits. Final Research Report EC BRITEEURAM MIDAS Project, p. 233 (1997).

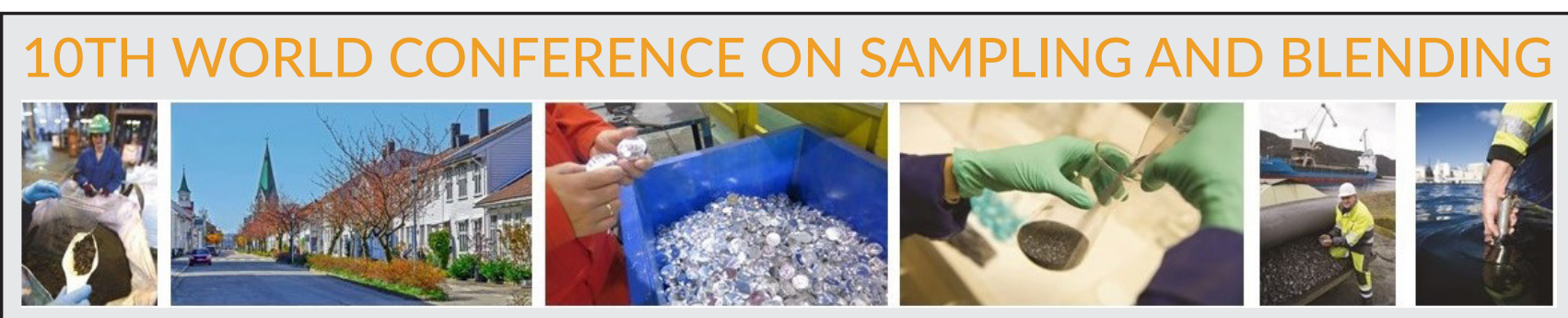

Correct sampling and analysis of raw materials are essential to ensure well-documented product quality and to contribute to a reduced environmental footprint. To this end, the WCSB10 conference covers the latest research and application experience of the Theory of Sampling and Blending.

31 May-2 June 2022, Kristiansand, Norway

\section{https://wcsb10.com}




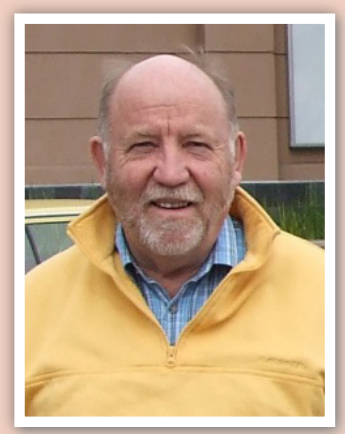

Dick Minnitt completed a MSc in geology in the Murchison Range and a PhD in the Richtersveld regions of southern Namibia. He joined Anglo American and later $\mathrm{JCl}$, after which he spent 14 years doing contract and consulting work. He completed a second MSc in mining, and joined the School of Mining Engineering at WITS in 1995, where he taught courses in Mineral Economics and Geostatistics. His interest in sampling of particulate materials arose from the numerous visiting lecturers he invited to Wits University including Dominique Francois Bongarçon, Francis Pitard, Geoff Lyman and Kim Esbensen. Dick retired from Wits in 2017, but continues to consult for international mining companies and research in his fields of interest. He now holds a position as a Visiting Emeritus professor where he continues to teach postgraduate classes and supervises masters and doctoral students.

(D) https://orcid.org/0000-0002-0267-8152

Richard.Minnitt@wits.ac.za

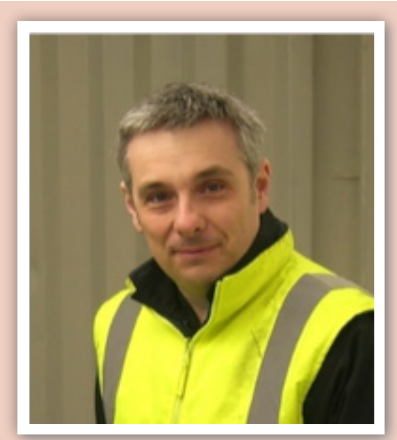

Dr Simon Dominy is a mining geologist-engineer with over 25 years based in operations, consulting and academia. He has experience across mine production, corporate business development, and multi-disciplinary studies. Simon has a background in underground operations management and technical/leadership roles, with multi-commodity and continent experience. He has worked across the mine value chain from project studies, through to mine reopening/ development, operations and operational improvement. He is a Visiting Associate Professor at the Camborne School of Mines, University of Exeter, UK, and holds technical roles with Novo Resources Corporation, Artemis Resources Ltd and OCX Gold Group.

(iD https://orcid.org/0000-0002-0638-3693

s.dominy@e3geomet.com

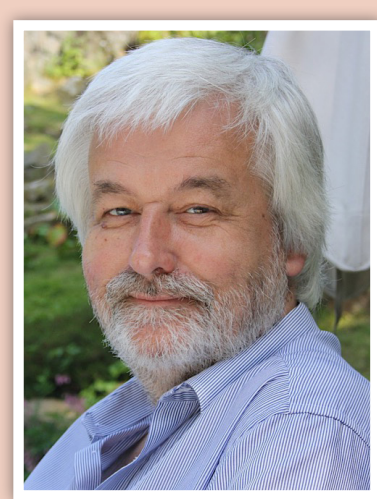

Kim H. Esbensen, PhD, Dr (hon), has been research professor in Geoscience Data Analysis and Sampling at GEUS, the National Geological Surveys of Denmark and Greenland (2010-2015), chemometrics \& sampling professor at Aalborg University, Denmark (2001-2015), professor (Process Analytical Technologies) at Telemark Institute of Technology, Norway (1990-2000 and 2010-2015) and professeur associé, Université du Québec à Chicoutimi (2013-2016). From 2015 he phased out a more than 30-year academic career for a new quest as an independent researcher and consultant. But as he could not terminate his love for teaching, he is still very active as an international visiting, guest and affiliate professor. A geologist/geochemist/metallurgist/data analyst of training, he has been working $20+$ years in the forefront of chemometrics, but since 2000 has devoted most of his scientific $R \& D$ to the theme of representative sampling of heterogeneous materials, processes and systems: Theory of Sampling (TOS), PAT (Process Analytical Technology) and chemometrics. He is a member of several scientific societies and has published over 250 peer-reviewed papers and is the author of a widely used textbook in Multivariate Data Analysis (35,000 copies), which was published in its $6^{\text {th }}$ edition in 2018. He was chairman of the taskforce behind the world's first horizontal (matrixindependent) sampling standard DS 3077 (2013). He is editor of the science magazine TOS forum and this Sampling Column. In 2020 he published the textbook: Introduction to the Theory and Practice of Sampling (impopen.com/sampling).

(iD) https://orcid.org/0000-0001-6622-5024

khe.consult@gmail.com 